\title{
SICHERHEITSPOLITIK DES MULTINATIONALEN UNTERNEHMENS
}

\author{
SECURITY POLICY OF MULTINATIONAL COMPANY
}

\author{
PATAKI János \\ (ORCID: 0000-0002-3387-5618) \\ bmp1k3@gmail.com
}

\begin{abstract}
Abstrakt
Bei der Erarbeitung der Sicherheitspolitik eines multinationalen Unternehmen müssen die Sicherheitsstrategie des betroffenen Landes und die Sicherheitsregelungen des Konzerns diese Regelungen sind "weltweit" gültig maximal beachtetet werden. Die Sicherheitspolitik des multinationalen Unternehmens ist das Grunddokument zur die Planung, Installation und Inbetriebnahmen der Sicherheits- und Verteidigungssysteme, die die Bestimmen des Vorstandes ihre mittel- und lange Sicherheitserwartungen und Sicherheitsvorschriften definiert wird.
\end{abstract}

Kernbegriffe: Sicherheitspolitik, multinationales Unternehmen, Nationale Sicherheitsstrategie

\begin{abstract}
The country's national security strategy and the "worldwide" security measures of a "mother" company in a multinational company must be fully taken into account when developing a company's security policy. The security and defence systems, the designing, construction and operation of the company's security strategy are basic documents that define the medium for the top management of the company and determine long-term safety requirements and regulations.
\end{abstract}

Keywords: security policy, multinational company, national security strategy 


\section{EINFÜHRUNG}

Die internationalen und amerikanischen Experten haben den NIC ${ }^{1}$-Bericht gemeinsam erstellt. Auf regionalen Konferenzen fassten sie die regionalen Probleme bzw. deren Auswirkungen auf die Trends mit globalem Effekt zusammen und werteten diese aus. Aus den acht erstellten Szenarien wurden vier ausgewählt, welche aus politischer und wirtschaftlicher Sicht am ehesten die denkbaren Ereignisse und Herausforderungen beleuchten. Diese Kapitel sind Szenarien, die ein mögliches Zukunftsbild skizzieren. Aus der Verwirklichung dieser Trends und den aufeinander ausgeübten Wirkungen entsteht das Weltbild für 2030.[1]

Die Bestimmungen und Schlussfolgerungen bezüglich der Sicherheitsumgebung des Multinationalen Unternehmens, der wichtigsten Bedrohungen und Risiken sind zutreffend, aber die Vorstellung der Sicherheitsstrategie der Nationalsicherheitsstrategie der Ungarn [2], bzw. auch die Vergleichung der Bestimmungen von beiden Strategien bezüglich der Bedrohungen.

\section{ZIELSETZUNG [3]}

Ziel der Sicherheitspolitik ist es, unter Berücksichtigung der Werte und Interessen, sowie auf Basis der Analyse des Sicherheitsumfeldes die Unternehmensziele, -aufgaben und -mittel zu bestimmen, durch die das Unternehmen in einer globalisierten Welt das Leben und die Gesundheit der Menschen bzw. das sichere Funktionieren des Unternehmens garantiert.

\section{Umfeld der Sicherheitspolitik}

Der Begriff der Sicherheit muss umfassend interpretiert werden, weil sich das Sicherheitsumfeld ständig verändert. Die globalen Herausforderungen, Drohungen, Gefahren und Risiken treten heute auf mehreren Ebenen auf; auf der Ebene der Individuen, Gemeinschaften, Staaten und Regionen und betreffen die Nichtregierungs- wie transnationale Organisationen.

Darum ist ein integrierter Umgang mit den sicherheitspolitischen, wirtschaftlichen und finanziellen, gesellschaftlichen (humanen) Dimensionen und Umweltaspekten von Bedeutung.

\section{Grundlage der Sicherheitsinteressen}

Am wichtigsten ist der Schutz des menschlichen Lebens und der Gesundheit. Bedeutsam ist zudem die Gewährleistung der Funktionsfähigkeit des Multinationalen Unternehmens, Überwachung und Schutz seiner Gesamtfläche, Einrichtungen und Vermögensgegenstände, Bewahrung ihrer inneren Ordnung und wirtschaftlichen Stabilität.

Der Vorstand des multinationalen Unternehmen erwartet, dass sich die Sicherheitsorganisationen so gut wie möglich den neuen Sicherheitsherausforderungen anpassen und möglichst angemessene Antworten auf die Gefahrenquellen geben.

\footnotetext{
${ }^{1}$ National Intelligence Council: GLOBAL TRENDS, PARADOX OF PROGRESS, Die Welt der nächsten zwanzig Jahre wird ausgesehen, präsentiert dieser einzigartige Zukunftsreport, den der Central Intelligence Agency (CIA) und der US-amerikanische National Intelligence Council (NIC) ausgearbeitet haben. Die globale Trends haben die folgende Punkte definiert:

1) Demographische Lage: die Reichen altern, die Armen nicht,

2) Die Weltwirtschaft verlagert sich,

3) Die Beschleunigung des technologischen Fortschritts,

4) Religiöse Glaubens und nationale Identitäten führen zu einer Welle von Diskriminierungen,

5) Das Regieren wird immer schwieriger,

6) Das Risiko von Konflikten (zwischen Staaten),

7) Der Klimawandel, Umweltkonflikte.
} 


\section{Sicherheitsgründe}

Jede Person, die sich im Zuständigkeitsbereich des multinationalen Unternehmens aufenthält, ist zur Einhaltung der Sicherheitsmaßnahmen verpflichtet.

Nach den Bestimmungen der Sicherheitsstrategie der multinationalen Unternehmens ist der Mitarbeiter mit Sicherheitsbewusstsein, der die Sicherheitsmaßnahmen annimmt, umsetzt und anwendet, ein grundlegender Faktor.

Das Fundament der Sicherheitspolitik ist die Zusammenarbeit der Mitarbeiter, Leiter, Interventionskräfte, Fachbereiche und der Geschäftsführung. Diese Zusammenarbeit bildet eine Wertegemeinschaft, die die Funktionsfähigkeit des multinationalen Unternehmens und die Sicherheit der Menschen, die mit ihm in Berührung kommen, garantiert.

Die beauftragten Fachbereiche arbeiten auf Basis der Sicherheitsstrategie und Sicherheitspolitik des multinationalen Unternehmens seine eigene jeweilige Fachpolitik aus, unterstützen seine Leiter durch Beratung bei der Verwirklichung der Sicherheitsmaßnahmen sowie bei der Beantwortung von unternehmensinternen Sicherheitsfragen.

\section{BEHANDLUNGEN DER BEDROHUNGEN [4]}

\section{Herausforderungen, Gefährden, Risiken}

Die Sicherheitsmaßnahmen müssen wissenschaftsbasiert und den aktuellen Schutzzielen entsprechend ausgearbeitet werden. Die Vorbereitung auf Notfälle umfasst die Vorbereitung der betroffenen Organisationen und die Koordination ihrer Zusammenarbeit sowie die Vorbereitung und Information der Mitarbeiter, ferner den Ausbau verschiedener Schutzfähigkeiten.

\section{Finanzkrise}

Die Frage der finanziellen Stabilität wurde als Folge der Weltwirtschaftskrise erheblich aufgewertet. Die Stabilität des wirtschaftlichen und finanziellen Systems und die Widerstandsfähigkeit des multinationalen Unternehmens sind ein wichtiges Interesse und ein wichtiger sicherheitspolitischer Aspekt. Das Risikomanagement bewertet kontinuierlich die aktuelle Lage, informiert den Vorstand und macht Vorschläge für das Krisenmanagement.

\section{Energiesicherheit}

Da die Energiesicherheit für das multinationale Unternehmen eine Grundsatzfrage ist, ist eine Diversifikation der Quellen und Lieferwege sowie des Einsatzes der Energieträger notwendig. Eine fehlende Diversifikation kann zahlreiche Risiken mit sich bringen. Wegen der Aufrechterhaltung der Funktionsfähigkeit des Unternehmens hat eine stabile, für einen Wettbewerbspreis erhältliche und planbare Energieversorgung hohe strategische Bedeutung.

\section{Klima- und Umweltwandel}

Der globale Klima- und Umweltwandel, die Auswirkungen der immer extremer werdenden Witterungsbedingungen, die Endlichkeit von Rohstoff- und Naturressourcen tragen ein ernsthaftes Sicherheitsrisiko in sich und können $\mathrm{zu}$ Konflikten führen. Wegen der geographischen Gegebenheiten der Region haben Umwelt- und Zivilisationsschäden, Überschwemmungen, Wasser- und Luftverschmutzung in den Nachbarländern des Karpatenbeckens eine verstärkte Auswirkung. Die Umweltgefahren wirken sich auf den Gesundheitszustand der Mitarbeiter und die Funktionsfähigkeit des Unternehmens indirekt aus. Daher sind die rechtzeitige Identifizierung der Gefahren und die enge Zusammenarbeit zwischen den Sicherheitsorganisationen des Unternehmens und den betroffenen staatlichen Behörden von großer Bedeutung. 


\section{Katastrophen}

Naturkatastrophen und industrielle Katastrophen sowie der Verlust der Kontrolle über die Prozesse in bestimmten industriellen, biologischen, chemischen und vor allem nuklearen Anlagen gefährden die menschliche Gesundheit, die Umwelt, die Sicherheit des menschlichen Lebens und Vermögens in besonderem Maße. Ein weiteres Risiko bedeutet der Transport von Gefahrgütern auf der Straße, auf Schienen, Wasserstraßen und Luftverkehrswegen sowie durch Rohrleitungen.

Das multinationale Unternehmen legt einen besonderen Wert auf die Aufrechterhaltung der kontinuierlichen Funktionsfähigkeit des Unternehmens, die effiziente Überwachung und den Schutz der Wirtschaft und der dazu notwendigen kritischen Infrastrukturen.

Im Interesse des Lebens- und Vermögensschutzes müssen insbesondere die entsprechende Vorbereitung der betroffenen Sicherheitsorganisationen und die Vorbereitung der Mitarbeiter für den Katastrophenfall gewährleistet werden.

\section{IT-Security ${ }^{2}$}

Durch die Cyber-Sicherheit müssen wir mit immer komplizierteren Herausforderungen im physischen und virtuellen Raum der IT- und Telekommunikationsnetzwerke sowie der damit verbundenen kritischen Infrastruktur rechnen. Erhöhtes Risiko bedeutet, dass die Ergebnisse der wissenschaftlichen und technologischen Entwicklung, die beinahe für alle zugänglich geworden sind, von bestimmten Staaten oder nichtstaatlichen Gruppen, sogar Terroristengruppen verwendet werden, um die Informations- und Kommunikationssysteme bzw. deren bestimmungsgemäße Funktion zu stören.

Vorrang genießen die folgenden Aufgaben: die regelmäßige Aufnahme und Priorisierung der im Cyberraum tatsächlich auftretenden potenziellen Bedrohungen und Risiken, Stärkung der Konzernkoordination, Erhöhung des Sicherheitsbewusstseins der Mitarbeiter und Verbesserung der Zusammenarbeit.

\section{Migration [5]}

Derzeit wird Ungarn von illegaler Migration in erster Linie als Transitland betroffen. Die sich aus der Komplexität der Lieferwege des Unternehmens ergebenden Aufgaben und die erfolgreiche Verwirklichung des erhöhten Schutzes der Außengrenzen setzen eine effizientere Zusammenarbeit der Organe, die an der Verwirklichung der ungarischen polizeilichen und mit der Migration verbundenen Aufgaben mitwirken, voraus. Ohne die Gewährleistung einer Markengruppenunterstützung ist es nicht zu erwarten, dass die betroffenen Organisationen gegen die Erscheinungsformen der illegalen Migration effizient auftreten können.

\section{Terrorismus [6]}

Die Bekämpfung des Terrorismus ist ein wichtiger Bereich. Sie bedeutet den Schutz der potenziellen Zielpunkte der Terroristen und die Erhöhung eigener Sicherheit, insbesondere die Sicherheit der Mitarbeiter bzw. der kritischen Infrastruktur des Unternehmens. Die rechtzeitige Prognose, Behinderung und Ausschaltung von Terrorangriffen ist die Aufgabe der staatlichen Sicherheitsorganisationen.

\footnotetext{
${ }^{2}$ IT-Sicherheit
} 


\section{INSTRUMENTE ZUR DURCHFÜHRUNG DER SICHERHEITSPOLITIK [7]}

Die Sicherheit des multinationalen Unternehmens muss auf festen wirtschaftlichen Grundlagen basieren, die die Ressourcen einer effizienten Sicherheitspolitik sichert. Das Unternehmen erachtet es für seine allfällige Aufgabe und Pflicht, im Einklang mit der wirtschaftlichen Situation entsprechende Ressourcen zum Schutz des Unternehmens und der Mitarbeiter bereitzustellen.

Ich halte es für wichtig, dass die Zusammenarbeit unter den Markengruppensicherheitsorganisationen, die bei der Aufrechterhaltung der Stabilität und Sicherheit eine entscheidende Rolle spielen, intensiver wird, und dass diese Organisationen eine einander stärkende und ergänzende Rolle spielen.

\section{Sicherheitsorganisationen}

Um die in der Sicherheitsstrategie des Unternehmens erscheinenden Bedrohungen umfassend zu behandeln, ist eine enge Zusammenarbeit der Fachbereiche notwendig.

Im Einklang damit müssen die enge und effiziente Zusammenarbeit und Koordination der Organisationen für Informations- und Datenschutz, Verteidigungsaufgaben, Feuerwehrtätigkeiten, Brandschutz-, Vermögens-, Katastrophenschutz und der bürgerlichen Organisationen für Krisenmanagement, sowie deren umfassenden, dem Sicherheitsumfeld angepassten Rahmen gestärkt werden. Jede Sicherheitsorganisation hat die Aufgabe, in ihrem Fachgebiet die Elemente der nationalen und internationalen Sicherheit und Bedrohungen ständig zu bewerten und die Schritte zu ihrer Bekämpfung und Abwehr einzuleiten. Das kann nur erfolgreich sein, wenn die Ressourcen der Organisationen koordiniert und konzentriert zur Anwendung kommen.

Die mit Sicherheit verbundene Tätigkeit der einzelnen Fachbereiche muss mit der Sicherheitsstrategie der Unternehmen im Einklang stehen. Während der periodischen Kontrolle der branchenspezifischen Fachpolitiken und Vorschriften muss man die einschlägigen Bestimmungen der Sicherheitsstrategie berücksichtigen.

Zur Umsetzung der Sicherheitspolitik und die Verteidigung der Interessen des Unternehmens sowie zur Wahrung der übergeordneten Unternehmensinteressen wurden von multinationalen Unternehmen „Minimum-Regelungen“ festgelegt.

Diese Ziele sind erreichbar, wenn die integrierten Sicherheitskonzepte und Ergänzungsmaßnahmen angewendet werden.

Die integrierten Sicherheitskonzepte werden durch technische, organisatorische und finanzielle Sicherheitsmaßnahmen geplant und realisiert.

\section{Sicherheitsregelwerk}

\section{Sicherheitsstrategie}

Die Sicherheitsstrategie muss vom Vorstand angenommen werden. Sie bedeutet die zielorientierte und integrierte Planung und Umformung der Sicherheitsmaßnahmen, die in ein nach dem Prinzip von ,check and balance” funktionierendes Sicherheitssystem eingebettet sind (die Sicherheitsverantwortung der Organisationseinheiten ist von den unterstützenden Fachbereichen und den Task Force ${ }^{3}$ getrennt).

\footnotetext{
${ }^{3}$ Werksfeuerwehr, Werkschutz, Rettungsdienst, Facility Management
} 


\section{Sicherheitsvorschriften / Regelungen}

Minimum-Regelungen und Sicherheitsvorschriften und -regel des multinationalen Unternehmens beziehen sich auf alle Organisationseinheiten:

- Sicherheitspolitik:

○ Arbeitssicherheitspolitik

○ Umweltschutzpolitik

○ Gesundheitspolitik

○ IT-Sicherheitspolitik.

- Informations- und Datenschutz-Regelungen ${ }^{4}$

- Objektschutz:

$\circ$ Zutrittsregelung

- Personenschutz

- Brandschutz

○ Vorbeugende Brandschutz

○ Werksfeuerwehr

- Krisenmanagement und $\mathrm{BCM}^{5}$

- Versicherungsschutz.

\section{ZUSAMMENFASSUNG}

Grundlegende sicherheitspolitische Ziele sind die fortdauernde Stärkung und Überwachung der wirtschaftlichen und finanziellen Stabilität, die rechtzeitige Aufklärung externen Gefährdungspotenzials, Begründung bzw. sichere und langfristige Stärkung der Funktionsfähigkeit des Unternehmens.

Die Sicherheitspolitik regelt die Anforderungen an die Sicherheitsorganisation des multinationalen Unternehmens sowie die Verantwortlichkeiten, Aufgaben und Maßnahmen zur Wahrung der Sicherheit.

Die Sicherheitspolitik dient dem Schutz der Unversehrtheit von Leib und Leben, des materiellen und geistigen Eigentums sowie dem Schutz der ungestörten Geschäftstätigkeit und der Reputation des multinationalen Unternehmens.

\section{LITERATURVERZEICHNIS}

[1] National Intelligence Council: Global Trends 2030: Paradox of Progress, https://www.dni.gov/files/documents/nic/GT-Full-Report.pdf $\quad$ (Heruntergeladen: 15.10.2018)

[2] Regierungsbeschluss Nummer 1035/2012. (II. 21.) über die Nationale Sicherheitsstrategie

[3] Managementhandbuch Sicherheitswirtschaft und Unternehmenssicherheit, (C) 2012 Richard Booberg Verlag, ISBN 978-3-415-04776-1, p. 95

[4] Col. István Resperger: A NEW APPROACH TO NATIONAL AND INTELNATIONAL CRISIS MANAGEMENT, Defence Review, Special Issue 2016/2, HU ISSN 2060-1506. pp. $215-228$

\footnotetext{
${ }^{4}$ Prototypenschutz, Geheimhaltungen, Fotoregelung ...

${ }^{5} \mathrm{BCM}$ - Business Continuity Management
} 
[5] Wörterbuch zur Sicherheitspolitik, Verlag E.S. Mittler \& Sohn, ISBN 3-8132-0797-8, 2003, p. 239

[6] Joachim Krause: Der „lange Krieg” des jihadistischen Terrorismus geht weiter, (C) 2014 Verlag Barbara Budrich, Opladen, Berlin \& Toronto, ISBN 978-3-8474-0115-5, pp. 15 24

[7] Managementhandbuch Sicherheitswirtschaft und Unternehmenssicherheit, (C) 2012 Richard Booberg Verlag, ISBN 978-3-415-04776-1, pp. 845 - 860

\title{
EGY MULTINACIONÁLIS VÁLLALAT BIZTONSÁGPOLITIKÁJA
}

\begin{abstract}
Absztrakt
Egy multinacionális vállalat biztonságpolitikájának kidolgozásánál maximálisan figyelembe kell venni az adott ország nemzeti biztonsági stratégiáját, valamint az „anya” vállalat „,worldwide” érvényes biztonsági intézkedéseit. A biztonsági és védelmi rendszerek tervezésénél, kiépitésénél és müködtetésénél a multinacionális vállalat biztonságpolitikája az az alap dokumentum, amelyben a felsö vezetés meghatározza vállalatra vonatkozó közép-és hosszú távú biztonsági elvárásait és elöírásait.
\end{abstract}

Kulcsszavak: biztonságpolitika, multinacionális vállalat, nemzeti biztonsági stratégia 